\title{
High Prevalence of Moyamoya Syndrome in Appalachia
}

\author{
Michael John Nisiewicz ${ }^{a} \quad$ Jill M. Roberts ${ }^{a, b}$ Michael R. Dobbs ${ }^{c}$ \\ Ebunoluwa A. Ajadi ${ }^{a}$ Patrick Kitzman ${ }^{d}$ Marc Wolfe $^{d}$ Kelley Elkins ${ }^{e}$ \\ Adam J. Dugan ${ }^{f}$ Justin F. Fraser ${ }^{a, b, g, h}$ \\ aDepartment of Neurological Surgery, University of Kentucky, Lexington, KY, USA; ${ }^{\mathrm{b}}$ Department of Neuroscience, \\ University of Kentucky, Lexington, $\mathrm{KY}, \mathrm{USA}$; ${ }^{\circ}$ Department of Neurology, University of Texas, Edinburg, TX, USA; \\ ${ }^{d}$ College of Health Sciences, University of Kentucky, Lexington, KY, USA; ${ }^{N}$ Norton HealthCare Stroke Network, \\ Lexington, KY, USA; 'Department of Biostatistics, University of Kentucky, Lexington, KY, USA; ${ }^{9}$ Department

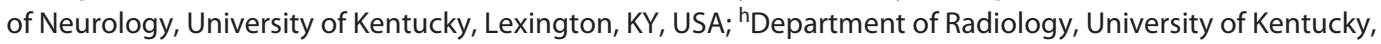 \\ Lexington, KY, USA
}

\section{Keywords}

Cerebrovascular disease - Moyamoya · Stroke · Prevalence

\begin{abstract}
Introduction: Moyamoya is a chronic cerebrovascular condition of unclear etiology characterized by progressive occlusion of 1 or both internal carotid arteries with neovascular collateral formation. With both an idiopathic form (moyamoya disease) and congenital condition-associated form (moyamoya syndrome), it can cause ischemic and hemorrhagic stroke. Recent findings in Kentucky have challenged traditional estimates of its incidence in US populations. Using the Kentucky Appalachian Stroke Registry (KApSR), our aim was to further characterize its incidence as a cause of stroke and to understand the patient population in Appalachia. Methods: A retrospective review of moyamoya patients was performed using the KApSR database. Data collected included demographics, county location, risk factors, comorbidities, and health-care encounters from January 1, 2012, to December 31, 2016. Results: Sixty-seven patients were identified; 36 (53.7\%) resided in Appalachian counties. The cohort accounted for 125 of 6,305 stroke admissions, representing an incidence of 1,983 per 100,000 stroke ad-
\end{abstract}

missions. Patients presented with ischemic strokes rather than hemorrhagic strokes (odds ratio 5.50, 95\% Cl: $2.74-$ $11.04, p<0.01)$. Eleven patients (16.4\%) exhibited autoimmune disorders. Compared to the general population with autoimmune disorder prevalence of $4.5 \%$, the presence of autoimmunity within the cohort was significantly higher ( $p<0.01)$. Compared to non-Appalachian patients, Appalachian patients tended to present with lower frequencies of tobacco use $(p=0.08)$, diabetes mellitus ( $p=0.13)$, and hypertension ( $p=0.16$ ). Conclusions: Moyamoya accounts for a substantial number of stroke admissions in Kentucky; these patients were more likely to develop an ischemic stroke rather than a hemorrhagic stroke. Autoimmune disorders were more prevalent in moyamoya patients than in the general population. The reduced frequency of traditional stroke risk factors within the Appalachian group suggests an etiology distinct to the population.

○ 2020 S. Karger AG, Basel

\section{Introduction}

Moyamoya is a chronic cerebrovascular condition of unclear etiology characterized by progressive occlusion of 1 or both internal carotid arteries, leading to ischemic and hem-

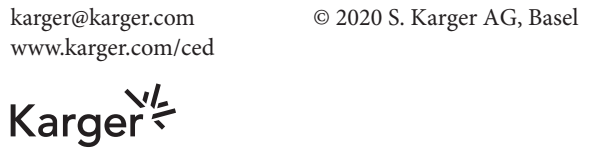

Justin F. Fraser

Departments of Neurological Surgery, Neurology, Radiology, and Neuroscience Center for Advanced Translational Stroke Science, University of Kentucky 800 Rose St., Room MS105B, Lexington KY 40536 (USA) jfr235@uky.edu 
Fig. 1. Age (a) and county distribution (b) of moyamoya patients in KY. Each numeral within a bordered region designates the number of moyamoya patients residing in that county. KY, Kentucky.

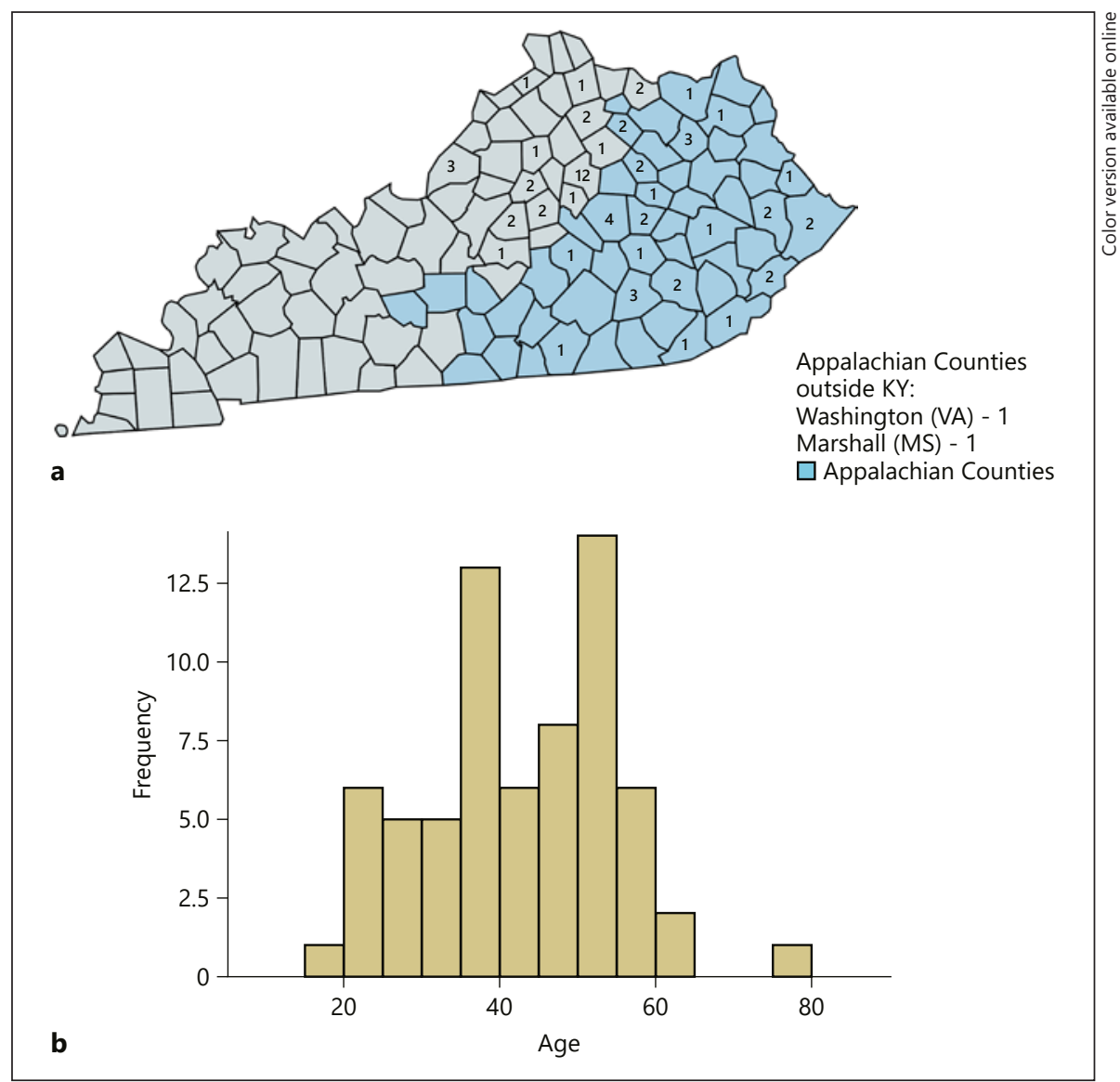

orrhagic strokes. Although initially characterized in Japan, incidence within various ethnic groups in other regions of the world has been evaluated [1-3]. Previous studies suggest that moyamoya has a low incidence within the USA [1], compared to the rates encountered in various regions of East Asia [2-4]. However, a recent analysis of moyamoya within Appalachia has suggested that its prevalence may significantly exceed prior US estimates [5]. To further evaluate this, we performed a retrospective review utilizing the Kentucky Appalachian Stroke Registry (KApSR) [6], to characterize its incidence as a cause of stroke and to characterize the patient population within our region.

\section{Methods}

The University of Kentucky Institutional Review Board reviewed and approved the study protocol. Due to the retrospective nature, the need for informed consent was waived. The data were gathered through KApSR, a data warehouse built from stroke-related data extracted from the University of Kentucky Health System, generating a "snapshot" dataset. KApSR is further described in a previous article [6]. Inclusion criteria were patients who presented from January 1, 2012, to December 31, 2016, and ICD-9 or ICD-10 codes for

Moyamoya Syndrome in Appalachia moyamoya. The variables collected included age, sex, race, county of residence, risk factors, comorbidities, number of health-care encounters, stroke type (ischemic or hemorrhagic), NIHSS score, and diagnosis at encounter. Patients were categorized as either Appalachian or non-Appalachian according to the county of residence. Counties were identified as Appalachian according to the designation by the Appalachian Regional Commission [7], and their associated populations were retrieved from US 2016 census records.

Patient characteristics and risk factors were compared by Appalachian status. For categorical variables, frequencies and column percentages (\%) were reported, and $p$ values were calculated using $\chi^{2}$ and Fisher's exact tests, as appropriate. For ordinal variables (the sums of risk factors), frequencies and column percentages (\%) were reported, and $p$ values were calculated using Mann-Whitney $U$ tests to account for ordinality. For continuous variables, $p$ values were calculated using Student's $t$ test. Composite risk factor variables were calculated from the following risk factors: hypertension, diabetes mellitus (type 1 or 2), and tobacco use. Statistical significance was set at $p \leq 0.05$.

\section{Results}

Sixty-seven patients with moyamoya were identified. The median age was 43 years, with a bimodal distribution at ages 35-39 and 50-54 years (Fig. 1a). Twenty-three 
Table 1. Moyamoya stroke admissions, 2012-2016

\begin{tabular}{|c|c|c|c|c|c|c|}
\hline Year & 2012 & 2013 & $2014^{\mathrm{a}}$ & 2015 & 2016 & Cumulative \\
\hline Total moyamoya admissions, $n(\%)$ & $12(1.0)$ & $13(1.1)$ & $32(2.5)$ & $35(2.6)$ & $33(2.5)$ & $125(2.0)$ \\
\hline Total stroke admissions & 1,211 & 1,150 & 1,301 & 1,311 & 1,332 & 6,305 \\
\hline Incidence of moyamoya per 100,000 stroke admissions & 991 & 1,330 & 2,460 & 2,670 & 2,477 & 1,983 \\
\hline
\end{tabular}

${ }^{a}$ Certification year of University of Kentucky Healthcare as a comprehensive stroke center.

Table 2. Patient characteristics and risk factors stratified by Appalachian status

\begin{tabular}{lccc}
\hline & $\begin{array}{c}\text { Appalachian } \\
(n=36)\end{array}$ & $\begin{array}{l}\text { Non-Appalachian } \\
(n=31)\end{array}$ & $p$ value \\
\hline Age, mean (SD), years & $41(11)$ & $44(14)$ & 0.335 \\
Sex, male, \% $n)$ & $36.1(13)$ & $32.3(10)$ & 0.741 \\
Race, white, \% $(n)$ & $100.0(36)$ & $71.0(22)$ & \\
Total number of encounters & 171 & 197 & 0.259 \\
Number of encounters, mean (SD) & $4.75(4.46)$ & $6.35(6.94)$ & 0.967 \\
Presenting NIHSS score, mean (SD) & $5.71(5.70)$ & $5.76(4.93)$ & 0.162 \\
Hypertension, \% $(n)$ & $44.4(16)$ & $64.5(20)$ & 0.083 \\
Tobacco use, \% $(n)$ & $25.0(9)$ & $48.4(15)$ & 0.206 \\
Autoimmunity, \% $(n)$ & $11.1(4)$ & $22.6(7)$ & 0.132 \\
Diabetes mellitus (T1/T2), \% ( $n)$ & $13.9(5)$ & $32.3(10)$ & 1,000 \\
Diabetes mellitus (T1), \% $(n)$ & $8.3(3)$ & $9.7(3)$ & 0.679 \\
Dyslipidemia, \% $(n)$ & $27.8(10)$ & $35.5(11)$ & 0.016 \\
Number of risk factors present (tobacco use, hypertension, and diabetes mellitus), $(\%) n$ \\
$\quad 0$ & $44.4(16)$ & $29.0(9)$ & \\
1 & $27.8(10)$ & $12.9(4)$ & \\
2 & $27.8(10)$ & $41.9(13)$ & \\
3 & 0 & $16.1(5)$ & \\
\hline
\end{tabular}

(34.3\%) patients were male. Racial profile was predominantly white, with a black minority. The cohort accounted for 368 encounters, of which 125 were stroke admissions. From 2012 to 2016, the cohort contributed 2.0\% $(n=125)$ of 6,305 stroke admissions (Table 1). Stroke diagnoses were predominantly ischemic $(n=49)$ rather than hemorrhagic $(n=9)$ (odds ratio 5.50, 95\% CI: $2.74-$ $11.04, p<0.01)$. Autoimmune disorders were observed in $16.4 \%(n=11)$ of the 67 patients (Table 2$)$, significantly higher than the general US population estimated prevalence of $4.5 \%(p<0.01)$ [7].

The Appalachian and non-Appalachian cohorts consisted of 36 and 31 patients, respectively. Distribution of patients according to the county of residence is depicted in Figure 1b. Both groups were comparable across admission and demographic data (Table 1), excluding race, whereby the non-Appalachian cohort comprised all 9 black patients. Utilizing 2016 Appalachian census populations ${ }^{9}$ and our cohort population, the calculated crude prevalence of moyamoya within the Appalachian territory of Kentucky was 2.91 cases per 100,000 versus non-Appalachians reporting 0.95 cases per $100,000(p<0.01)$. Prevalence per county is presented in Tables 3 and 4. Appalachians and non-Appalachians did not differ in the number of admissions per patient nor the NIHSS admission score. Tobacco use ( 49.4 vs. $25.0 \%, p=0.08$ ), diabetes mellitus ( 32.3 vs. $13.9 \%, p=0.13$ ), and hypertension ( 64.5 vs. $44.4 \%, p=$ 0.16 ) were more frequent in non-Appalachian patients but did not meet the threshold for statistical significance. With the consideration of cumulative presence of these 3 previous risk factors, the overall frequency was significantly higher in non-Appalachian than Appalachian patients ( 1.45 vs. $0.83, p=0.02)$. Autoimmunity and dyslipidemia were similar in both groups (Table 2). Autoimmune disorders included type 1 diabetes, systemic lupus erythematosus, immune thrombocytopenic purpura, and Addison's disease. 
Table 3. Kentucky Appalachian counties, moyamoya prevalence, and population growth

\begin{tabular}{lcl}
\hline County & $\begin{array}{c}\text { Prevalence } \\
\text { per } 100,000(2016)\end{array}$ & $\begin{array}{l}\text { Population growth } \\
(2012-2016), \%\end{array}$ \\
\hline Bell & 3.69 & -3.7 \\
Breathitt & 7.53 & -3.2 \\
Carter & 3.7 & -1.3 \\
Clay & 9.63 & -4.1 \\
Estill & 13.98 & $-1,3$ \\
Floyd & 5.39 & -5.5 \\
Harlan & 3.68 & -5.8 \\
Jackson & 7.48 & +0.4 \\
Laurel & 4.98 & +0.9 \\
Letcher & 8.78 & -5.3 \\
Lewis & 7.44 & -2.2 \\
Lincoln & 4.10 & -0.1 \\
Madison & 4.47 & +4.9 \\
Marion & 5.21 & -3.9 \\
Martin & 8.33 & -6.2 \\
Montgomery & 7.20 & +3.0 \\
Nicholas & 28.23 & +1.0 \\
Pike & 3.30 & -6.5 \\
Powell & 8.12 & -1.8 \\
Rowan & 12.27 & +2.7 \\
Wayne & 4.89 & -0.5 \\
All counties & 2.91 & -1.3 \\
\hline & &
\end{tabular}

${ }^{a}$ Includes Appalachian counties without identified moyamoya patients.

\section{Discussion}

The purpose of this study was to assess the admission rates and features of patients exhibiting moyamoya within our region. As observed in previous analyses, our cohort demonstrated a predominance of female patients [8] but differed significantly with respect to race. While Asian descent is recognized to have a significant association with the development of moyamoya [1], this study involved a predominantly white patient group reflective of the regional population. With only 67 patients, the cohort accounted for $2 \%$ of stroke admissions, emphasizing the frequency that this condition is encountered within Kentucky. However, with the certification of our institution as a comprehensive stroke center during 2014, there was a notable increase in the frequency of moyamoya presentations. Additionally, the Appalachian moyamoya estimated prevalence is comparable to previous studies in East Asia [2-4], despite the absence of Asian patients, but these numbers remain only half of what has been observed in Japan [9]. It should be noted that the presenta-
Table 4. Kentucky non-Appalachian counties, moyamoya prevalence, and population growth

\begin{tabular}{|c|c|c|}
\hline County & $\begin{array}{l}\text { Prevalence } \\
\text { per 100,000 (2016) }\end{array}$ & $\begin{array}{l}\text { Population growth } \\
\text { (2012-2016), \% }\end{array}$ \\
\hline Anderson & 9.03 & +2.1 \\
\hline Bourbon & 4.99 & +0.4 \\
\hline Fayette & 3.77 & +4.5 \\
\hline Franklin & 1.98 & +1.7 \\
\hline Harrison & 10.73 & -0.3 \\
\hline Jefferson & 0.39 & +2.1 \\
\hline Jessamine & 1.91 & +5.5 \\
\hline Marion & 5.21 & -3.9 \\
\hline Mason & 11.63 & -1.6 \\
\hline Mercer & 9.31 & +0.5 \\
\hline Pendleton & 6.87 & +0.2 \\
\hline Washington & 16.52 & +2.6 \\
\hline All counties ${ }^{\mathrm{a}}$ & 0.95 & +3.3 \\
\hline
\end{tabular}

${ }^{a}$ Includes non-Appalachian counties without identified moyamoya patients.

tions observed within this study likely identify moyamoya syndrome, compared to most East Asian analyses that consider moyamoya disease. The values observed within this study underestimate the prevalence as a result of the limitations of single comprehensive stroke center network data and accessibility to health-care services. With other in-state and nearby out-of-state providers, the estimates proposed within this study are likely underrepresentative. However, while we have started to screen moyamoya patients at our institution with a full battery of autoinflammatory markers, with nearly all screened moyamoya patients exhibiting the syndromic variant, this process was not performed for this cohort at the time of data collection. Nonetheless, we believe that this cohort consisted primarily of the syndromic variant.

Moyamoya was found to be significantly more frequent in Appalachians than non-Appalachians. This finding is encouraged by the greater size of the Appalachian cohort within a population of 1.2 million, in comparison to the 3.3 million within the non-Appalachian territory [7]. Migration presents a limit to our analysis, given that the migratory patterns of families and patients could not be determined. However, from 2012 to 2016, the Appalachian population exhibited a contraction of $1.3 \%$ (Table 3 ) compared to a $3.3 \%$ growth in the nonAppalachian territory (Table 4). The relative prevalence is also complicated by both the racial profile and the relative frequency of risk factors between the 2 cohorts. De- 
spite the higher frequency of traditional stroke risk factors and the significant number of African-American patients, the Appalachian cohort still exhibited a higher prevalence. Given these counterintuitive findings, it is possible that there is an etiology of moyamoya distinct to this population.

There has yet to be an identified etiology of adult-onset moyamoya syndrome. However, the pathophysiology likely involves an autoimmune phenomenon. Our results further support the association of moyamoya with autoimmunity, as the frequency of known autoimmune diseases within our cohort was significantly higher than expected within the general US population [10]. A similar association was observed in another midwestern US state by Bower et al. [11] who studied their institutional population in Minnesota. The authors noted a startling $>20$ fold difference in the prevalence of autoimmunity, although they compared their moyamoya cohort to the institutional general population prevalence. Genetic analyses have also identified distinct markers that associated with moyamoya. A case-control study in Japan reviewed human leukocyte antigen (HLA) genotypes of moyamoya patients. The authors noted that the frequencies of the HLA-DRB1 allele were elevated in moyamoya patients compared to that in the control, and moyamoya patients with this allele expressed increased frequencies of Graves' disease and thyroiditis [12]. Furthermore, moyamoya patients have been observed to express elevated serum levels of (s)CD163, an activating marker for CD163+ macrophages, which has been implicated in numerous autoimmune conditions [13]. Despite repeated observation of this autoimmune phenomenon, the pathogenesis remains unidentified.

There are limitations to performing a study using coding databases. The data abstractions are limited to recognized and diagnosed conditions that are cited in the medical record. Furthermore, due to the de-identified nature of the data extraction process, we were limited in our ability to examine each patient's medical record individually. However, institutionally, our standard workflow for the evaluation of moyamoya includes digital subtraction angiography, which is the gold standard for diagnosis. Although all stroke patients within our institution undergo head and neck CTA imaging, we do not rely on noninvasive imaging to make the diagnosis, so the coding diagnoses are unlikely to overrepresent the incidence. If anything, our data, being limited to our stroke network, underestimates the prevalence of the disease, as other moyamoya patients may have received care through other hospital networks. This further supports our conclu- sions that Appalachians have a higher than previously thought prevalence of moyamoya.

\section{Conclusions}

Moyamoya accounts for a disproportionate number of stroke admissions within the Kentucky region, predominantly exhibiting an ischemic course. Autoimmunity is a common comorbidity in these patients, consistent with previous reports, exceeding expected population frequencies. The Appalachian territory appears to exhibit a higher prevalence of the condition, despite lower frequencies of traditional risk factors relative to non-Appalachian patients, which may be suggestive of an etiology distinct to this population.

\section{Acknowledgements}

The study described was supported by the NIH National Center for Advancing Translational Sciences through Grant No. UL1TR001998. The content is solely the responsibility of the authors and does not necessarily represent the official views of the NIH.

\section{Statement of Ethics}

This study was approved by the University of Kentucky Institutional Review Board (IRB-45668). Due to the retrospective nature, the need for informed consent was waived.

\section{Conflict of Interest Statement}

All authors have no conflicts of interest to disclose.

\section{Author Contributions}

M.J.N. contributed to data collection, manuscript writing, manuscript editing, and figure design. M.R.D. contributed to study conception, study supervision, and manuscript editing. J.M.R. contributed to study supervision, manuscript writing, and manuscript editing. E.A.A. contributed to manuscript writing and figure design. P.K., M.W., and K.E. contributed to development of KApSR and data collection. A.J.D. contributed to manuscript writing and statistical analysis. J.F.F. contributed to study conception, manuscript writing, manuscript editing, and study supervision. 


\section{References}

1 Uchino K, Johnston SC, Becker KJ, Tirschwell DL. Moyamoya disease in Washington State and California. Neurology. 2005;65(6):956-8.

2 Miao W, Zhao PL, Zhang YS, Liu HY, Chang $\mathrm{Y}, \mathrm{Ma}$ J, et al. Epidemiological and clinical features of moyamoya disease in Nanjing, China. Clin Neurol Neurosurg. 2010;112(3):199203.

3 Chen PC, Yang SH, Chien KL, Tsai IJ, Kuo MF. Epidemiology of moyamoya disease in Taiwan: a nationwide population-based study. Stroke. 2014;45(5):1258-63.

4 Wakai K, Tamakoshi A, Ikezaki K, Fukui M, Kawamura T, Aoki R, et al. Epidemiological features of moyamoya disease in Japan: findings from a nationwide survey. Clin Neurol Neurosurg. 1997;99(Suppl 2):S1-5.

5 Wang CY, Grupke SL, Roberts J, Lee J, Fraser JF. Factors associated with moyamoya syndrome in a kentucky regional population. J Stroke Cerebrovasc Dis. 2018;27(3):793-800.
6 Kitzman P, Wolfe M, Elkins K, Fraser JF, Grupke SL, Dobbs MR. The kentucky appalachian stroke registry (KApSR). J Stroke Cerebrovasc Dis. 2018;27(4):900-7.

7 Pollard K, Jacobsen LA. The appalachian region: a data overview from the 2012-2016 American community survey chartbook. 2018.

8 Mejia-Munne JC, Ellis JA, Feldstein NA, Meyers PM, Connolly ES. Moyamoya and inflammation. World Neurosurg. 2017;100: 575-8.

9 Kuriyama S, Kusaka Y, Fujimura M, Wakai K, Tamakoshi A, Hashimoto S, et al. Prevalence and clinicoepidemiological features of moyamoya disease in Japan: findings from a nationwide epidemiological survey. Stroke. 2008;39(1):42-7.
10 Hayter SM, Cook MC. Updated assessment of the prevalence, spectrum and case definition of autoimmune disease. Autoimmun Rev. 2012;11(10):754-65.

11 Bower RS, Mallory GW, Nwojo M, Kudva YC, Flemming KD, Meyer FB. Moyamoya disease in a primarily white, midwestern US population: increased prevalence of autoimmune disease. Stroke. 2013;44(7):1997-99.

12 Tashiro R, Niizuma K, Khor SS, Tokunaga K, Fujimura M, Sakata $\mathrm{H}$, et al. Identification of HLA-DRB $1 * 04: 10$ allele as risk allele for Japanese moyamoya disease and its association with autoimmune thyroid disease: a casecontrol study. PLoS One. 2019;14(8): e0220858.

13 Fujimura M, Fujimura T, Kakizaki A, SatoMaeda M, Niizuma K, Tomata Y, et al. Increased serum production of soluble CD163 and CXCL5 in patients with moyamoya disease: involvement of intrinsic immune reaction in its pathogenesis. Brain Res. 2018;1679: $39-44$. 